\title{
EXPERIMENTAL DETERMINATION OF OHMIC RESISTIVITY OF FLY ASH FOR DESIGN OF ELECTROSTATIC PRECIPITATORS
}

\begin{abstract}
M. Ozawa, ABSTRACT
and J. I. Yanagihara ${ }^{a}$

Laboratory of Energy \& Thermal Engineering

Department of Mechanical Engineering

Escola Politécnica - University of São Paulo

Av. Prof. Mello Moraes, 2231

CEP 05508-900, São Paulo/SP, Brasil

ajiy@usp.br

The objective of the present work is to determine the ohmic resistivity of fly ashes collected at electrostatic precipitators (ESPs) attached to boilers burning Brazilian coals. An experimental device to measure the fly ash resistivity was designed and constructed; tests were conducted according to accepted standards in other countries. It was the first time that this kind of measurement was conducted for Brazilian coals. The tested materials were collected from ESPs operating in the southern region of Brazil, where the mineral coal is commonly used. The ohmic resistivity of the tested materials was smaller compared to mineral coals from abroad. The resistivity of the fly ash increased when the temperature was raised, which is in accordance with reported results for mineral coals from abroad. Tests with particulate material collected from the electrostatic precipitator (ESP) of a biomass boiler showed that its ohmic resistivity is much lower than the resistivity of fly ash from the mineral coal. This means that the ESP for biomass boiler should be smaller, which is in accordance with the industrial practice. Based on the experimental results, practical comments about ESP sizing were made in order to assist engineers and technicians dealing with the problem of gas treatment in coal combustion.
\end{abstract}

\section{NOMENCLATURE}

$a$

A

$A_{d}$

$E_{e}$

$E_{p}$

$E \quad$ Electrical field, $\mathrm{V} / \mathrm{m}$

$F_{D} \quad$ Viscous drag force, $\mathrm{N}$

$F_{E} \quad$ Electrostatic force, $\mathrm{N}$

I Electrical current, A

$J_{A, \mathrm{j}} \quad$ Electrical current density, $\mathrm{A} / \mathrm{m}^{2}$

$S C A$ Specific collecting area, $\mathrm{m}^{2} / \mathrm{m}^{3} / \mathrm{s}$

$T$ Temperature, ${ }^{\circ} \mathrm{C}$

$t$ Thickness of dust layers, $\mathrm{mm}$ or $\mathrm{cm}$

$V \quad$ Total gas flow rate, $\mathrm{m}^{3} / \mathrm{s}$

$V^{g} \quad$ Voltage, $\mathrm{V}$

$w \quad$ Migration velocity, $\mathrm{cm} / \mathrm{s}$

\section{Greek symbols}

$\varepsilon_{o} \quad$ Electrical permissivity of air, F/m

$\kappa \quad$ Dielectric constant of air

$\mu \quad$ Dynamic viscosity of air, $\mathrm{kg} / \mathrm{m} . \mathrm{s}$

$\eta \quad$ Collecting efficiency, $\%$

$\rho, \rho_{A}$ Resistivity, ohm.cm

$\rho_{g} \quad$ Gas resistivity, ohm.cm

$\rho_{d} \quad$ Resistivity of dust, ohm.cm
Keywords: electrostatic precipitator, ohmic resistivity, mineral coal, fly ash

\section{INTRODUCTION}

Coal is widely used in fired boilers in many countries because of its abundance and low cost. On the other hand, due to environmental concerns nowadays, its use as energy source requires the utilization of many environmental protection equipments to treat the combustion gases.

Brazilian coal is very cheap and a large quantity of coal is available in the southern region of Brazil. However, it has a high amount of ash and due to this fact, its combustion in boilers produces pollution in the gas stream (e.g. dust, particulate material). These ashes must be collected and adequately disposed of in order to lower its impact on the environment. Electrostatic precipitators are commonly used to solve this kind of problem and its use should increase from now on.

On the other hand, technology regarding electrostatic precipitators used in Brazil comes from abroad. The design constraints were established by using the physical properties of the foreign coal. It is important that Brazilian engineers and technicians were able to design, manufacture, install and conduct performance tests on site, with reliable technical information about the precipitation process and the characteristics of the coal.

In Brazil, there are few research works focusing on electrostatic precipitators. Particularly, there are no references addressing the ohmic 
characteristic of fly ash of mineral coals.

The aim of this study is to evaluate the ohmic resistivity of fly ash from mineral coals used in Brazil. The ohmic resistivity of the fly ash is an important design parameter because it provides information for the engineer to design the electrostatic precipitator, defining its size and specific collecting area in order to achieve the aimed performance. It also allows the engineer to predict the electric behavior of electric collecting fields, to calculate the existing electrostatic precipitator efficiency and to evaluate the quantity of outlet particulate material after the electrostatic precipitator.

\section{ELECTROSTATIC PRECIPITATOR (ESP)}

An electrostatic precipitator is a device that removes particles from a gas stream. It accomplishes particle separation by using an electric field that imparts a negative charge to the particle, which is attracted to an oppositely charged plate or tube. The particles are removed from the collecting plate to a hopper by vibrating or rapping the collecting plate.

From the equilibrium between electrostatic force $\left(F_{E}\right)$ and drag force $\left(F_{D}\right)$ applied in the particle, it is possible to calculate the migration velocity of the particle (relative to the gas stream) $w$, assuming the Stokes' dragging.

$$
\begin{array}{r}
F_{D}=6 \pi \mu a w=4 \pi \varepsilon_{o} p c \\
p=\left[2 \cdot\left(\frac{\kappa-1}{\kappa+2}\right)+1\right]
\end{array}
$$

where $\mu$ is the air viscosity, $a$, the particle radius, $\varepsilon_{o}$, the electrical permissivity of air, $\kappa$, the dielectrical constant of air, and $E_{e}$ and $E_{p}$ are the electrical fields for electrification and precipitation, respectively. From Eq. (1), the migration velocity can be calculated as:

$$
w=\frac{2 p \varepsilon_{o} E_{e} E_{p} a}{3 \mu}
$$

Considering the migration velocity $w$, the volumetric gas flow rate $V_{g}$ and the total collecting area $A$, the expression below may be deduced to estimate the precipitator efficiency (White, 1962):

$$
\eta=1-\varepsilon^{-A \cdot c o / \varsigma_{\gamma}}
$$

It is noteworthy that the precipitator efficiency is a function of the particle size.

\section{OHMIC RESISTIVITY OF PARTICLE}

For the flying particles to deposit on the collecting surface of precipitators, they must possess a certain value of electrical conductivity in order to conduct the ionic current from the corona discharge to the ground. Very high conductivity leads to a loose filter cake, favoring re-entrainment of already precipitated dust. On the other hand, very low conductivity produces a strong voltage drop across the filter cake. This not only weakens the electrical field in the channel but may also lead to the so called "back-corona", which means electrical discharges in the filter cake. This produces ions of opposite polarity than the corona, neutralizing the particles and thus preventing them from being precipitated.

The minimum value of electrical conductivity, as found by theory and experience, is around $10^{-10}(\mathrm{ohm} . \mathrm{cm})^{-1}$. From the practical point of view, this value of conductivity is comparable to that of common metals, but it is larger than the conductivity of electrical insulating materials. Particles with the electrical conductivity lower than the critical value of $10^{-10}$ (ohm.cm) $)^{-1}$ are known as particles of high resistivity.

The ohmic resistivity is defined by Eq. (5):

$$
\rho=\frac{E}{j}
$$

where $\rho$ is the ohmic resistivity, $E$ is the electrical field between electrodes and $j$ is the electrical current density.

The operation of precipitators with high resistivity particles is usually followed by instability of the electrical condition. It may produce excessive sparking in high voltage or excessive current in low voltage. These effects reduce the collecting efficiency of the precipitator (decrease of particulate collecting). When the resistivity increases above $10^{11} \mathrm{ohm}-\mathrm{cm}$, it becomes too difficult to achieve reasonable efficiency with 
conventional precipitators. In this case, special types of ESP must be used or, more commonly, measures should be taken to reduce the resistivity.

\section{RESISTIVITY AS ESP DESIGN PARAMETER}

Figure 1 shows the effect of the resistivity of the particle on the migration velocity of ESPs (White, 1962). The results were obtained from a pilot experiment in laboratory scale with cement particulate. It is clearly noticeable that the migration velocity of the particle decreases from $15 \mathrm{~cm} / \mathrm{s}$ at $10^{10} \mathrm{ohm}-\mathrm{cm}$ to just $3 \mathrm{~cm} / \mathrm{s}$ at $10^{11} \mathrm{ohm}-$ $\mathrm{cm}$. The particle resistivity can be modified by the addition of humidity or some chemical components in the gases or in the fly ashes.

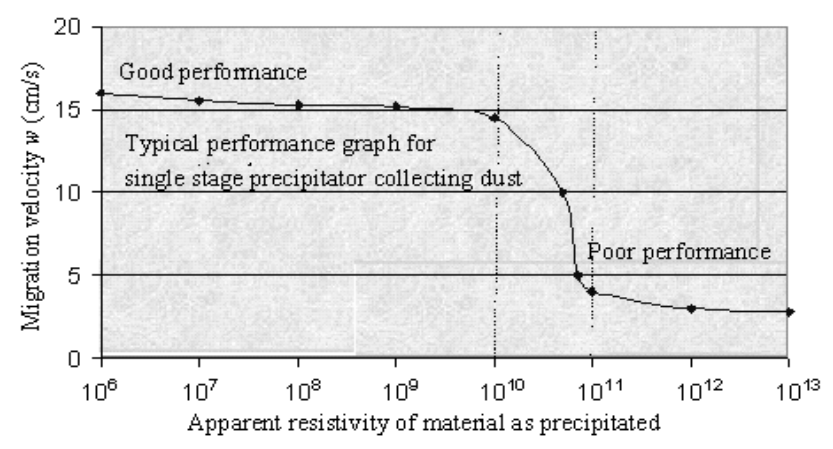

Figure 1. Effect of particle resistivity on ESP performance (White, 1962)

Suzuki and Tachibana (1984) burnt 30 types of coals in a pilot plant and measured the precipitability of the fly ashes produced. It was found that sulfur has a major effect on the fly ash collecting efficiency in a cold ESP (ESP with temperature lower than $250^{\circ} \mathrm{C}$ ). The precipitability of fly ash increases when there is injection of $\mathrm{SO}_{3}$, depending on the kind of coal.

Chae and Seo (1998) studied the improvement of ESP performance by pulse energization in order to collect fly ashes with high resistivity and a very fine dust. The supply of an appropriate electric pulse, according to the dust characteristic and design of ESP, can produce a high collecting performance.

Fujishima and Tsuchiya (1998) developed a cold type ESP for coal-fired boiler with high efficiency. The technique consists in placing the ESP downstream a heat exchanger, reducing the gas temperature to around $90^{\circ} \mathrm{C}$. As a result, the dust resistivity is reduced and the collecting efficiency increases.
Jedrusik et al. (2003) conducted a study to investigate the influence of the physicochemical properties (chemical composition, particle size distribution and resistivity) of the fly ash on the collection efficiency. They concluded that an increased content of sulphur compounds $\left(\mathrm{SO}_{3}\right)$ and sodium compounds $\left(\mathrm{Na}_{2} \mathrm{O}\right)$ reduced the resistivity and raised the sorbing activity of the fly ash, thus increasing wettability as well as adhesion forces and cohesion between the fly ash particles. The agglomerates formed in this way were found to be easier to remove.

\section{EXPERIMENTAL METHOD AND DEVICE}

The experimental apparatus used for the conductivity measurement is shown in Fig. 2. It consists of a small plate that encloses a quantity of fly ash and serves as the negative pole, called the discharge electrode. Another plate serves as the positive pole, where the particles are attracted to and is called the collecting electrode. The basic test cell and the test species are placed inside an insulated box with an electrical heating element in order to keep the temperature constant.

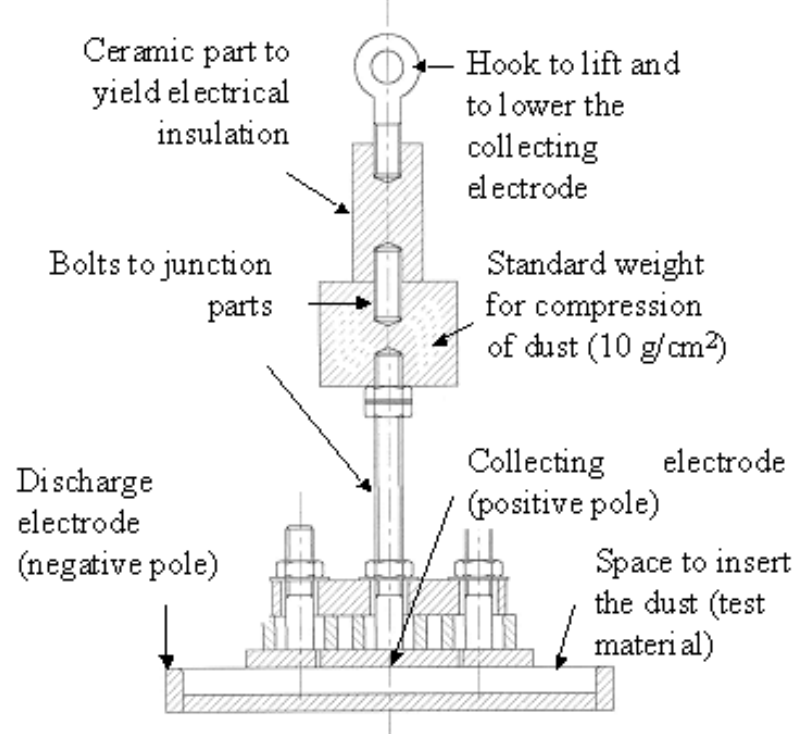

Figure 2. Description of experimental apparatus

The dust is submitted to an increasing voltage $V[\mathrm{~V}]$ and the breakdown current $I[\mathrm{~A}]$ is measured just before the electrical sparking occurs. The ohmic resistance of dust $R$ [ohm] is calculated by these two measured parameters, as shown in Eq. (6). 


$$
R=\frac{V}{I}
$$

The resistivity $\tilde{n}$ is calculated by Eq. (7):

$$
\rho=R \cdot \frac{A_{d}}{t}
$$

in which $A_{d}\left(\mathrm{~cm}^{2}\right)$ is the area of the disc where the dust is deposited and $t(\mathrm{~cm})$ is the thickness of the dust layer.

The experimental procedure consists of the following steps. The lower plate (discharge electrode) is filled with the dust that forms a uniform layer. The upper plate (collecting electrode) is placed on the dust layer. The dust compactness is defined by the upper plate mass, which has a standard weight. The temperature is varied assuming three distinct values (ambient temperature, $\mathrm{T}=50^{\circ} \mathrm{C}$ and $\mathrm{T}=70^{\circ} \mathrm{C}$ ) and the humidity is measured by a wet bulb thermometer. The electrical voltage is increased slowly $(500 \mathrm{~V}$ each step) and the start of the back corona or the formation of glow on dust layer is detected. The breakdown voltage can be estimated or determined by direct measurement. This standard technique for measuring the resistivity is described in the ASME PTC 28 document - Determination of Proprieties of Particulate Material. The test cell was constructed according to this standard. The experimental data (voltage $V$ and current $I$ ) was taken when sparking or electrical breakdown occurred. The electrical voltage breakdown could be observed by four different ways: due to the instability of the electrical supply voltage control; due to the instability of the electrical current indicator; due to a visible electrical discharge, as voltaic arc; and due to the typical noise from an electrical discharge. After three measurements for the same sample, the value of the voltage $V$ was estimated to be 0.95 of the average breakdown voltage. The experimental data obtained with this procedure had great repeatability.

\section{RESULTS AND DISCUSSION}

In this experiment, fly ashes from four different sources were tested: fly ashes collected in a ESP at CGTEE (Companhia de Geração Térmica de Energia Elétrica), fly ashes collected in a ESP at COPESUL (Companhia Petroquímica do Sul), mineral coal used by CGTEE and the dust from a biomass boiler.

For the CGTEE's fly ash, the collecting process begins at the voltage of $1425 \mathrm{~V}$ (electrical field of $2850 \mathrm{~V} / \mathrm{m}$ ), which represents about $40 \%$ of the electrical breakdown voltage. In the experiment, due to the continuous electrical supply, the dust adheres strongly to the collecting electrode. As the transformer rectifier used in the experiment was limited to $6 \mathrm{kV}$, the smaller particles were collected first, and the larger particles were kept in the discharge electrode (lower plate). The largest particles do not move to the collecting electrodes because the weight of the particle is higher than the electrostatic force. So, at an initial stage, the dust is separated by granulometry.

The part of dust considered unburned $(0.10 \%$ to $3.42 \%$ - carbon rich fragments, with typical black color) is collected first. It means that the dust with a larger amount of unburned content is easier to be collected, because the fly ash layer conducts ions easily. An undesirable effect of the combustion process (the incomplete combustion in boiler) favors the fly ashes collecting process. This result agrees well with the conclusions of Potter (1984) and Shibuya and Mochizuki (1984).

The experimental results presented in Fig. 3 shows that the ohmic resistivity increases with temperature (temperature range: ambient temperature to $70^{\circ} \mathrm{C}$ ). The surface conductivity is predominant for low temperature and wet conditions. This result agrees with the published results for fly ashes of mineral coals from other countries (Oglesby and Nichols, 1978). The CGTEE's fly ash has a lower resistivity compared to some fly ash from abroad. It is possible to say that back corona, due to the high resistivity of fly ash, will not occur in this case, what favors the electrostatic precipitation process and the precipitator sizing. The CGTEE's fly ash can be classified as dust with low or medium ohmic resistivity. The ohmic resistivity for the experiment with smaller dust thickness is larger and is more sensitive to the temperature change (Fig. 3). This may be explained by the geometrical factors affecting the relative dust compactness.

By comparing materials from different sources (Fig. 4), it was observed that the dust from biomass boilers have much smaller resistivity 
compared to the fly ash from other mineral coal. This is in accordance with the industrial practice, as the ESP of biomass boilers are smaller than the ESP of coal fired boilers, considering the same volumetric gas flow rate. This occurs because the dust of biomass boilers has high sulfur content (from wood chips) and, consequently, low resistivity. The CGTEE's fly ash has lower resistivity than the COPESUL fly ash and higher resistivity than the own coal that produces the fly ash.

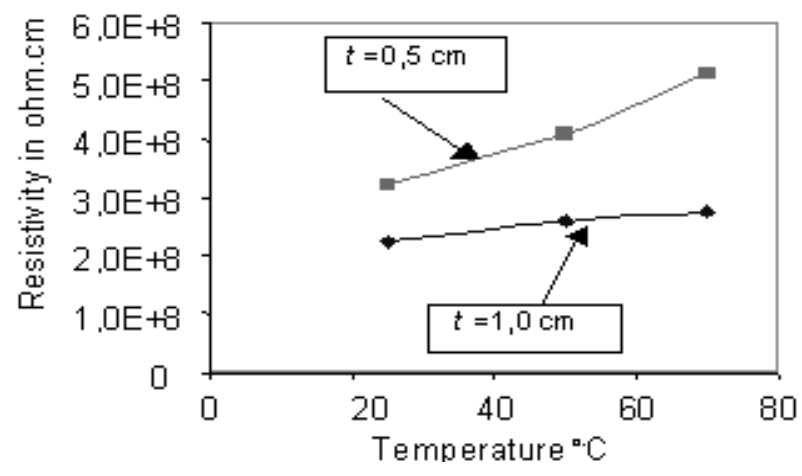

Figure 3. Influence of temperatura on resistivity

Analyzing the chemical composition of the CGTEE's coal, the content of $\mathrm{Fe}_{2} \mathrm{O}_{3}$ ranges from $6 \%$ to $10.60 \%$. Dalmon (1984) verified that this amount of $\mathrm{Fe}_{2} \mathrm{O}_{3}$ favors the precipitation process due to an increase of the effective migration velocity. The sulfur content is medium, from $1.52 \%$ to $2.18 \%$, which also favors the collection of dust, as described by Suzuki and Tachibana (1984). The surface resistivity increases with the reduction of the sulfur content in the coal (Moleshi, 1983). Concerning the $\mathrm{CaO}$ content $(0.63 \%$ to $0.92 \%)$, the CGTEE's coal can be classified as low calcium content $(\mathrm{CaO}<2.5 \%)$.

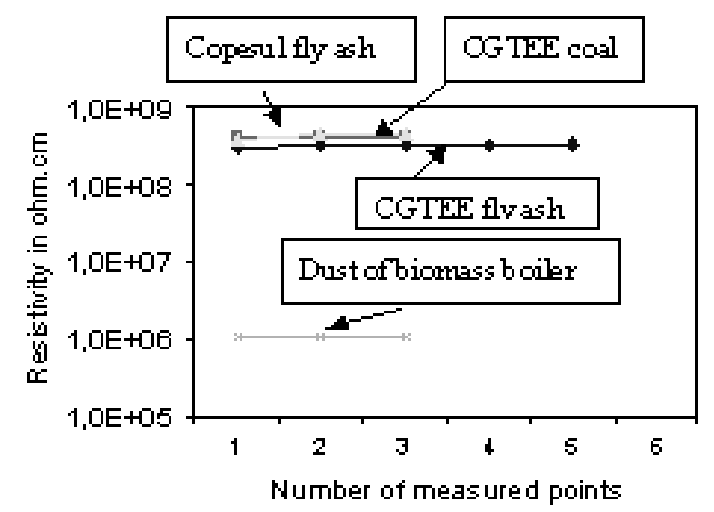

Figure 4. Comparison for different materials

Uncertainty analysis was carried using the methodology described in the ASME PTC 19.1 document - Measurement Uncertainty. The main sources of uncertainty were the evaluations of area and dust thickness. The relative uncertainty obtained for the ohmic resistivity measurement was $10 \%$, for $95 \%$ of confidence level.

\section{CONCLUSIONS}

An experimental device for fly ash resistivity measurement was designed and constructed; tests were conducted according to accepted standards in other countries. The tested materials were obtained from industrial sites that use Brazilian coals. It was the first time that this kind of measurement was conducted for Brazilian coals.

The CGTEE's fly ash does not present high ohmic resistivity, with values ranging from $3.04 \times 10^{8}$ to $5.35 \times 10^{8} \mathrm{ohm} . \mathrm{cm}$. The collecting process starts at a low voltage, indicating that due to the particle's low resistivity, the ESP can collect the fly ash at normal electrical voltage. Chemicalconditioning agents are not necessary as resistivity reducers.

The resistivity of the CGTEE's fly ash increases when the temperature increases. This trend is in agreement with the measurement results for mineral coals from abroad. As the fly ash of the CGTEE does not have high resistivity $\left(\rho>10^{11}\right.$ ohm.cm), it is expected that the back corona effect should not occur. When electrical sparking happens, the collecting process of dust is interrupted and a small cloud of dust is formed. This effect is undesirable to the industrial electrostatic precipitation process as the efficiency decreases and the dust goes out from the stack.

The tests with particulate materials from ESP of a biomass boiler showed that their ohmic resistivities are much lower than the resistivity of the Brazilian mineral coal. It means that the ESP for biomass boiler should be smaller, which is in accordance with the industrial practice.

he low ohmic resistivity means, from the practical point of view, that the migration velocity is higher and therefore, smaller ESPs are required. The Brazilian fly ash has low to medium resistivity and is likely to lack the undesirable back corona effect. Therefore, the ohmic resistivity should not be a key design concern for ESP attached to boilers burning Brazilian mineral coals. The design engineer must pay attention to other design parameters such as the internal velocity of gases 
and how to deal with the excess of fly ash content in the coal (up to $50 \%$ ).

\section{REFERENCES}

Chae, J.O., and Seo K.W., 1998, A Study of ESP Using Pulse Energization for Collecting High Resistivity and Fine Particle, In: 7th International Conference on Electrostatic Precipitation, Kyongju, Korea, pp. 375-382.

Dalmon, J., 1984, Ash Chemistry, Resistivity and Electrostatic Precipitator Performance, In: 2nd International Conference on Electrostatic Precipitation, Kyoto, Japan, pp. 299-309.

Fujishima H., and Tsuchiya, S., 1998, Colder Side ESP of Advanced Flue Gas Treatment System for Coal Fired Boiler, In: 7th International Conference on Electrostatic Precipitation, Kyongju, Korea, pp.542-549.

Jedrusik, M., Swierczok A., and Teisseyre, R., 2003, Experimental Study of Fly Ash Precipitation in a Model Electrostatic Precipitator with Discharge Electrodes of Different Design, Powder Technology, Vols. 135-136, pp. 295-301.

Moleshi, B., 1983, Electromechanics and Electrical Breakdown of Particulate Layers, Doctoral Thesis, University of Stanford, Palo Alto, CA

Oglesby, S., and Nichols, G. B., 1978, Electrostatic Precipitation, Marcel Dekker Inc.

Potter, E. C., 1984, Dust resistivity - Its Significance and Interpretation, In: 2nd International Conference on Electrostatic Precipitation, Kyoto, Japan, pp. 228-235.

Shibuya, S., and Mochizuki, Y., 1984, Evaluation of ESP Precipitability of Coal Fly Ash using Laboratory Combustor, In: 2nd International Conference on Electrostatic Precipitation, Kyoto, Japan, pp. 805-811.

Suzuki, J., and Tachibana, N., 1984, Precipitability of Fly Ash from Various Coals, In: 2nd International Conference on Electrostatic Precipitation, Kyoto, Japan, pp.194-199.

White, H., 1962, Industrial Electrostatic Precipitation, Addison-Wesley Publishing Company. 